\title{
Ampullitis Superimposed Obstructive Jaundice in a Patient With COVID-19
}

\author{
Can $\mathrm{Cao}^{\mathrm{a}}$, Renuka Lakshminarayanan ${ }^{\mathrm{b}}$, John McCracken ${ }^{\mathrm{c}}$, Jinping Lai ${ }^{\mathrm{b}, \mathrm{d}}$
}

\begin{abstract}
While coronavirus disease 2019 (COVID-19) is well known to cause significant lower respiratory symptoms, recent literature has documented numerous cases of multi-systemic involvement that can present with atypical symptoms. We report a case of an 83-year-old man, recovering from abdominal aortic aneurysm repair complicated by colonic injury requiring colostomy rendering him dependent on gastrostomy tube feedings for 3 years, who was transferred from a nursing care facility to the emergency department with altered mental status, fever and jaundice. Abdominal imaging and biopsy studies eventually identified duodenitis and ampullitis complicated by a suspected Klatskin tumor leading to biliary obstruction, sepsis and hepatoencephalopathy. Polymerase chain reaction (PCR) for COVID-19 was positive. Despite the severity of the initial presentation, the patient had no respiratory symptoms or abnormal chest X-ray findings on admission and developed hypoxia late into the disease course. Thus, this case is a report of an abnormal initial COVID-19 presentation with gastrointestinal and hepatobiliary involvement leading to hepatoencephalopathy but no lung findings, highlighting the importance of investigating extrapulmonary processes in COVID-19-positive patients regardless of pulmonary symptoms.
\end{abstract}

Keywords: COVID-19; Duodenitis; Ampullitis; Hepatoencephalopathy; Biliary intraepithelial neoplasia; Endoscopic retrograde cholangiopancreatography; Biliary brushing and cytopathology

\section{Introduction}

Beginning late 2019, severe acute respiratory syndrome co-

Manuscript submitted December 9, 2020, accepted January 20, 2021

Published online February 19, 2021

aThe Warren Alpert Medical School, Brown University, Providence, RI, USA bepartment of Pathology and Laboratory Medicine, Kaiser Permanente Sacramento Medical Center, Sacramento, CA, USA

'Division of Gastroenterology, Kaiser Permanente Sacramento Medical Center, Sacramento, CA, USA

${ }^{\mathrm{d} C o r r e s p o n d i n g ~ A u t h o r: ~ J i n p i n g ~ L a i, ~ D e p a r t m e n t ~ o f ~ P a t h o l o g y ~ a n d ~ L a b o r a t o r y ~}$ Medicine, Kaiser Permanente Sacramento Medical Center, Sacramento, CA 95825, USA. Email: Jinping.X.Lai@kp.org

doi: https://doi.org/10.14740/gr1353 rona virus 2 (SARS-CoV-2) rapidly disseminated around the world, leading to the current coronavirus disease 2019 (COVID-19) pandemic that caused over 2 million deaths globally [1]. While the respiratory manifestations are hallmark to the disease, multi-system involvement has been implicated in a significant number of cases [2,3]. Recent reports have shown that both the gastrointestinal (GI) tract and the liver are susceptible to COVID-19, leading to GI symptoms and abnormal liver function tests (LFTs) [4-9]. Herein we report an unusual case of COVID-19 presenting initially with symptoms in the duodenum, ampulla and hepatobiliary systems but without signs or symptoms of respiratory involvement until late in the disease progression.

\section{Case Report}

An 83-year-old man with history of hypertension, osteoarthritis and abdominal aortic aneurysm repair 3 years ago complicated by colonic injury requiring colostomy and percutaneous endoscopic gastrostomy presented with acute onset altered mental status, jaundice and right arm weakness. On arrival, the patient was febrile to $102.5^{\circ} \mathrm{F}$, tachycardic, and tachypneic but did not require supplemental oxygen given an oxygen saturation of $96 \%$ on room air. Initial labs were significant for blood white cell count $(10,400 / \mu \mathrm{L})$ with $84 \%$ of neutrophils, elevated lactic acid $(3.5 \mathrm{mmol} / \mathrm{L})$ and multiple abnormal LFTs (alanine aminotransferase (ALT) $617 \mathrm{U} / \mathrm{L}$, aspartate transaminase (AST) $376 \mathrm{U} / \mathrm{L}$, total bilirubin $5.4 \mathrm{mg} / \mathrm{dL}$ and alkaline phosphatase $452 \mathrm{U} / \mathrm{L}$ ), pointing toward severe sepsis with acute organ dysfunction. Initial chest X-ray (CXR) and computed tomography (CT) scan of the head identified no acute processes, ruling out potential stroke. The patient tested positive for COVID-19 by PCR (per nasopharyngeal swab) 1 day prior to admission as a resident of a local nursing home with an active outbreak. However, given the unremarkable CXR at presentation and lack of respiratory symptoms, the positive result was considered an incidental finding.

Ultrasound of the upper right abdominal quadrant revealed biliary dilatation and tumefactive sludge, leading to a magnetic resonance cholangiopancreatography (MRCP) study that confirmed significant biliary obstruction. Later, the patient underwent endoscopic retrograde cholangiopancreatography (ERCP) to directly visualize the region showing duodenal major papillae ulceration with ampullary involvement (Fig. 1). A metal biliary stent was placed to alleviate the obstruction in the 

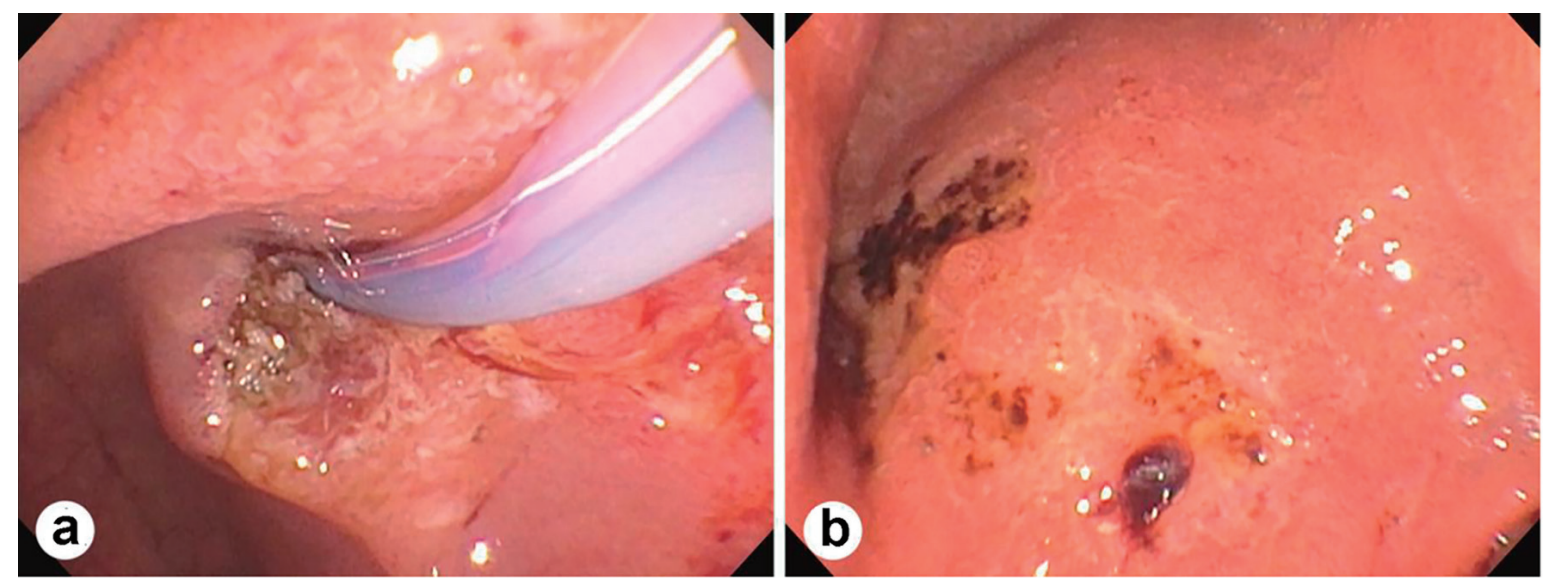

Figure 1. Endoscopic retrograde cholangiopancreatography (ERCP) showing duodenal major papillae and ampullary ulceration (a) and multiple ulcers in the duodenal bulb with bleeding (b).

distal common bile duct, and brushing samples were obtained around the stricture once dilated. Additionally, multiple duodenal ulcers causing upper GI bleeds were identified, and biopsies were obtained from ulcers on the ampulla and duodenal bulb. No significant mass lesions were identified by imaging or ERCP. Post-stenting, good bile flow was restored and the patient became afebrile soon after.

Microscopically, the morphology and immunohistochemistry of the biopsies were consistent with mucosal ulceration and acute inflammation associated with subepithelial vasculitis, thrombi formation, possible viral cytopathy with smudge nuclei and myenteric plexus involvement (Fig. 2). No carci-
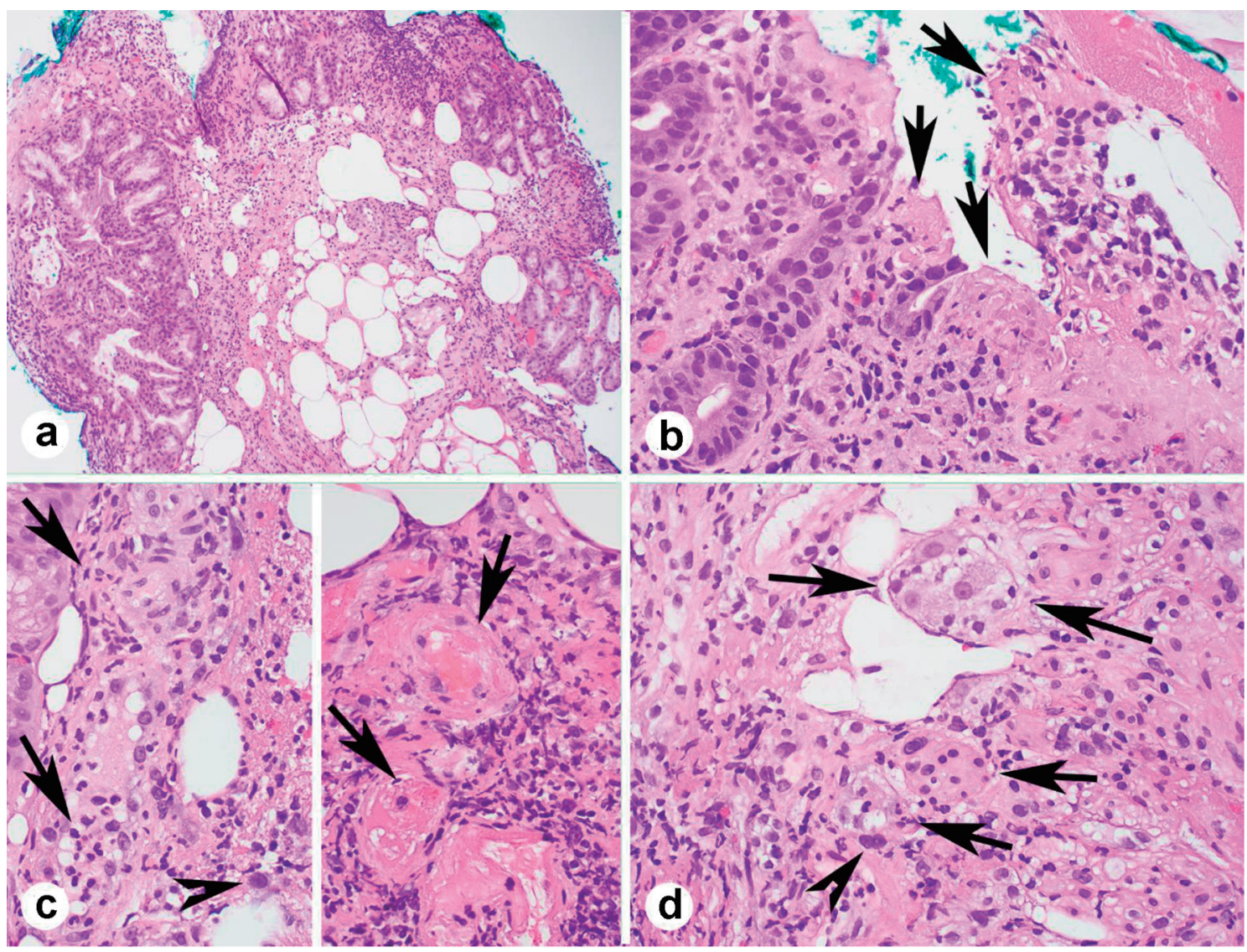

Figure 2. Duodenal major papillae and ampullary biopsy showing mucosal ulceration (a, b, arrows), vasculitis (c, left, arrows), thrombi (c, right arrows), smudge nuclei (c, left, and d, arrow heads) and ganglions involved by the inflammation (d, arrows) (a-d, H\&E stains; a, × 100; b-d, × 400). 


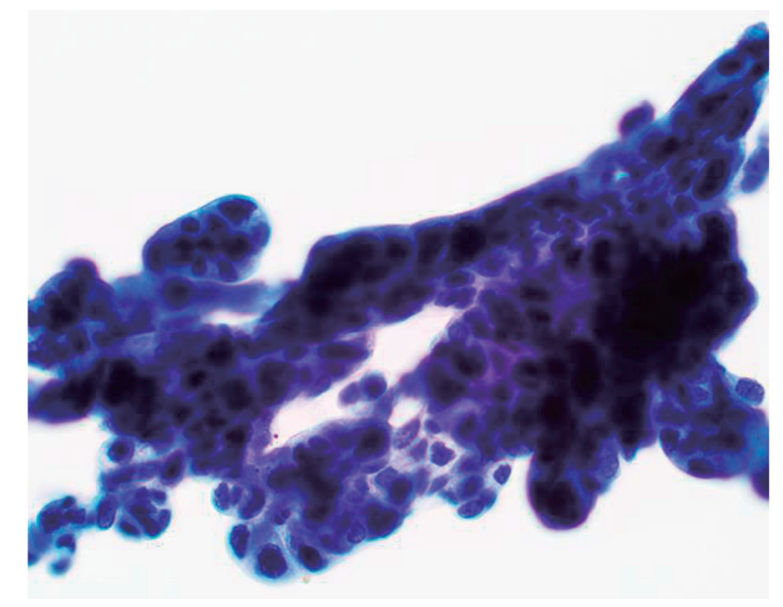

Figure 3. Bile duct brushing specimen showing clusters of atypical epithelial cells with high-grade dysplasia (Papanicolaou stain, $\times 400$ ).

noma or dysplasia was identified in either biopsy sample. The bile duct brushing sample contained rare clusters of atypical epithelial cells suspicious for high-grade dysplasia (Fig. 3). Cumulatively, the pathology was suggestive of two intersecting processes: first, an underlying suspected Klatskin tumor sparring the ampulla and second, a superimposed possible COVID-19 infection leading to worsening biliary stricture, ascending cholangitis, sepsis and hepatoencephalopathy.

On day 8 of admission ( 9 days after testing positive for COVID-19), the patient remained afebrile but developed progressive dyspnea and hypoxia complicated by hypernatremia from poor oral intake. CXR taken was still unremarkable. Dexamethasone, supplemental oxygen and fluid boluses were administered. Due to his acute kidney injury, hepatic insufficiency and suspected malignancy, the patient was not a candidate for remdesivir or convalescent plasma (CCP). The patient continued to decline despite intervention. Repeat CXR on day 13 of admission ( 2 weeks after testing positive for COVID-19) revealed mild hazy density within the lower lung lobes bilaterally without focal consolidation (Fig. 4). Later on day 13, the patient developed severe tachycardia, hypotension and hypoxia refractory to $100 \%$ non-rebreather therapy. Comfort care was initiated on day 14 of admission, and the patient expired shortly after.

\section{Discussion}

In recent COVID-19 case reviews and organoid studies, SARSCoV-2 has been shown to cause GI symptoms secondary to global inflammation and to directly infect GI tract and hepatic ductal tissues, which both express the angiotensin converting enzyme 2 (ACE2) receptor key to viral entry [4, 10-12]. GI symptoms such as loss of appetite, diarrhea, nausea and vomiting were reported in up to a third of COVID-19 patients [4$6,13,14]$. Inflammatory bowel diseases (IBDs) are a group of immune and inflammatory diseases and patients with IBD seem to be more vulnerable to influenza and COVID-19 [7, 8]. Evidence shows that COVID-19 can exacerbate symptoms of

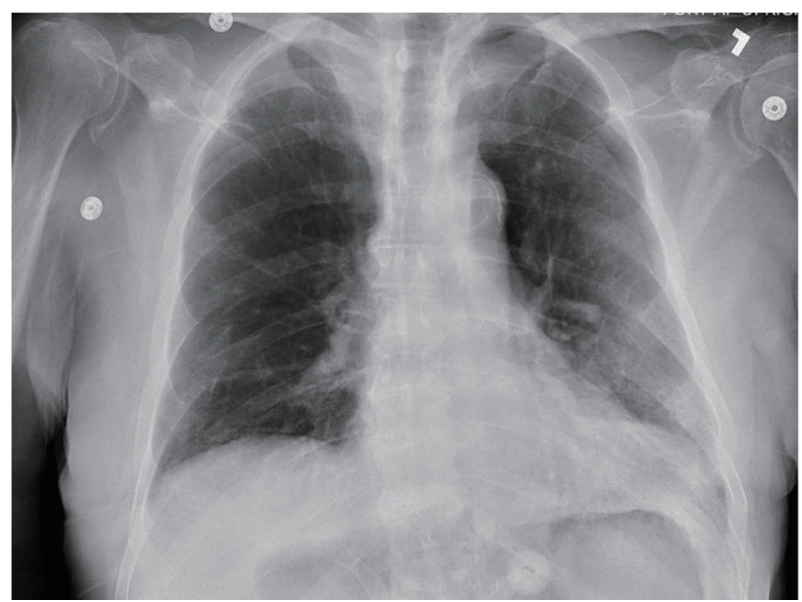

Figure 4. Chest X-ray showing mild hazy density within the lower lung lobes bilaterally without focal consolidation.

IBD and it is important to distinguish between an IBD exacerbation and symptoms caused by COVID-19 [9]. Patients with active severe IBD and with COVID-19 can go through progressive pneumonia, acute respiratory distress syndrome and multi-organ failure due to the cytokine storm syndrome associated with hyper-inflammation [9]. Liver injury, seen primarily through abnormal LFTs and bilirubin elevations, was reported in $14.8-50 \%$ of all patients $[14,15]$. Of note, GI and hepatic signs and symptoms were present in over half of patients with severe disease and have been associated with higher rates of hospitalization [4, 14].

Current estimates of the prevalence of GI COVID-19 manifestations in the absence of respiratory symptoms is less than $10 \%$ of cases [5]. The lack of respiratory involvement in this reported case is particularly unusual because of the severity of the initial presentation. While the most cohesive diagnosis for this patient was a duodenal/ampullary infection superimposed on a potential underlying biliary malignancy, it is possible that the ampullitis was a coincidental finding to the COVID-19 diagnosis or was due to another underlying infection or inflammatory process. Recent literature hypothesized that fecal-oral transmission of SARS-CoV-2 may be possible given that many patients have been shown to shed viral RNA into feces for weeks after clinical recovery $[6,11]$. While currently unproven, this alternate transmission route may explain how some patients do not present with pulmonary manifestations until late into the disease course.

Additionally this case highlights the importance of identifying extrapulmonary manifestations of COVID-19 in patients even without pulmonary involvement. Because the patient had an unremarkable CXR and denied respiratory symptoms for the first part of his admission, the positive result was considered initially incidental. Given that the possible clinical presentations and treatments of COVID-19 are ever-expanding, thorough investigation into positively testing patients is critical to both preparation for potential COVID-19-related sequelae and successful treatment.

In summary, this report documents a case of COVID-19 with an initial presentation of duodenal and ampullary acute 
inflammation leading to ascending cholangitis, sepsis and altered mental status/hepatoencephalopathy. To our knowledge, this is the first such case of severe extrapulmonary GI-hepatobiliary involvement occurring in the absence of respiratory symptoms until late into the disease course. Clinicians and patients alike should be vigilant for atypical COVID-19 manifestations given the current unknowns regarding the disease.

\section{Acknowledgments}

None to declare.

\section{Financial Disclosure}

None to declare.

\section{Conflict of Interest}

None to declare.

\section{Informed Consent}

Informed consent was obtained.

\section{Author Contributions}

CC wrote the manuscript; RL made the pathologic diagnosis; JM collected the data; JL made the diagnosis, collected and analyzed the data, and finalized the manuscript.

\section{Data Availability}

Any inquiries regarding supporting data availability of this study should be directed to the corresponding author.

\section{References}

1. WHO coronavirus disease (COVID-19) dashboard. Accessed Jan 26, 2021. https://covid19.who.int/.

2. Gavriatopoulou M, Korompoki E, Fotiou D, Ntanasis-Stathopoulos I, Psaltopoulou T, Kastritis E, Terpos E, et al. Organ-specific manifestations of COVID-19 infection. Clin Exp Med. 2020;20(4):493-506.
3. Zhang Y, Geng X, Tan Y, Li Q, Xu C, Xu J, Hao L, et al. New understanding of the damage of SARS-CoV-2 infection outside the respiratory system. Biomed Pharmacother. 2020;127:110195.

4. Mao R, Qiu Y, He JS, Tan JY, Li XH, Liang J, Shen J, et al. Manifestations and prognosis of gastrointestinal and liver involvement in patients with COVID-19: a systematic review and meta-analysis. Lancet Gastroenterol Hepatol. 2020;5(7):667-678.

5. Pan L, Mu M, Yang P, Sun Y, Wang R, Yan J, Li P, et al. Clinical characteristics of COVID-19 patients with digestive symptoms in Hubei, China: a descriptive, cross-sectional, multicenter study. Am J Gastroenterol. 2020;115(5):766-773.

6. Han C, Duan C, Zhang S, Spiegel B, Shi H, Wang W, Zhang L, et al. Digestive symptoms in COVID-19 patients with mild disease severity: clinical presentation, Stool Viral RNA Testing, and Outcomes. Am J Gastroenterol. 2020;115(6):916-923.

7. Altamimi E. Effect of COVID-19 pandemic and lockdown on children with gastrointestinal disorders. Gastroenterology Res. 2020;13(3):125-128.

8. Ahmed M. Coronavirus Disease 2019: A Gastroenterologist's Perspective in May 2020. Gastroenterology Res. 2020;13(3):89-95.

9. Barbalho SE, Matias JN, Flato UAP, et al. What do influenza and COVID-19 represent for patients with inflammatory bowel disease? Gastroenterology Res. 2021;14(1):112.

10. Tan HW, Xu YM, Lau ATY. Angiotensin-converting enzyme 2: The old door for new severe acute respiratory syndrome coronavirus 2 infection. Rev Med Virol. 2020;30(5):e2122.

11. Lamers MM, Beumer J, van der Vaart J, Knoops K, Puschhof J, Breugem TI, Ravelli RBG, et al. SARSCoV-2 productively infects human gut enterocytes. Science. 2020;369(6499):50-54.

12. Lin L, Jiang X, Zhang Z, Huang S, Zhang Z, Fang Z, $\mathrm{Gu} Z$, et al. Gastrointestinal symptoms of 95 cases with SARS-CoV-2 infection. Gut. 2020;69(6):997-1001.

13. Machhi J, Herskovitz J, Senan AM, Dutta D, Nath B, Oleynikov MD, Blomberg WR, et al. The natural history, pathobiology, and clinical manifestations of SARS-CoV-2 infections. J Neuroimmune Pharmacol. 2020;15(3):359386.

14. Thuluvath PJ, Alukal JJ, Ravindran N, Satapathy SK. What GI physicians need to know during COVID-19 pandemic. Dig Dis Sci. 2020.

15. Xu L, Liu J, Lu M, Yang D, Zheng X. Liver injury during highly pathogenic human coronavirus infections. Liver Int. 2020;40(5):998-1004. 